\title{
Antipsychotic drug use in pregnancy: high dimensional, propensity matched, population based cohort study
}

\author{
Simone N Vigod, ${ }^{1,2,3}$ Tara Gomes, ${ }^{2}$ Andrew S Wilton, ${ }^{2}$ Valerie H Taylor, ${ }^{1,3}$ Joel G Ray ${ }^{2,4}$
}

'Women's College Research

Institute; Department of

Psychiatry, University of

Toronto, Toronto, Ontario M5S

1B2, Canada

2Institute for Clinical Evaluative Sciences, Toronto, Ontario M4N 3M5

${ }^{3}$ Department of Psychiatry, Women's College Hospital; University of Toronto, Toronto, Ontario

${ }^{4}$ Departments of Medicine and Obstetrics and Gynaecology, St Michael's Hospital, University of Toronto, Toronto, Ontario M5B 1W8

Correspondence to: S N Vigod simone.vigod@wchospital.ca

Additional material is published online only. To view please visit the journal online (http://dx.doi. org/10.1136/bmj.h2298)

Cite this as: BMJ 2015;350:h2298 doi: 10.1136/bmj.h2298

Accepted: 22 March 2015

\begin{abstract}
OBJECTIVE

To evaluate maternal medical and perinatal outcomes associated with antipsychotic drug use in pregnancy. DESIGN

High dimensional propensity score (HDPS) matched cohort study.

SETTING

Multiple linked population health administrative databases in the entire province of Ontario, Canada.

PARTICIPANTS

Among women who delivered a singleton infant between 2003 and 2012, and who were eligible for provincially funded drug coverage, those with $\geq 2$ consecutive prescriptions for an antipsychotic medication during pregnancy, at least one of which was filled in the first or second trimester, were selected. Of these antipsychotic drug users, 1021 were matched 1:1 with 1021 non-users by means of a HDPS algorithm.
\end{abstract}

\section{MAIN OUTCOME MEASURES}

The main maternal medical outcomes were gestational diabetes, hypertensive disorders of pregnancy, and venous thromboembolism. The main perinatal outcomes were preterm birth ( $<37$ weeks), and a birth weight $<3$ rd or $>97$ th centile. Conditional Poisson regression analysis was used to generate rate ratios and $95 \%$ confidence intervals, adjusting for additionally prescribed non-antipsychotic psychotropic medications.

RESULTS

Compared with non-users, women prescribed an antipsychotic medication in pregnancy did not seem to

\section{WHAT IS ALREADY KNOWN ON THIS TOPIC}

Antipsychotic drug use in pregnancy has increased in recent years, but most studies have evaluated the older, "typical" antipsychotic drugs, which are now less commonly used

The few studies that have evaluated atypical antipsychotic drugs in pregnancy suggest that these drugs may be associated with increased risk for maternal metabolic complications in pregnancy (such as gestational diabetes) with resultant consequences (such as altered fetal growth), but these are limited in terms of sample size and in considering important confounding factors

\section{WHAT THIS STUDY ADDS}

Our study suggests that antipsychotic drug use in pregnancy does not independently increase risk for important short term maternal medical and perinatal outcomes

This is largest study of antipsychotic exposed pregnancies to date and the only study that used a high dimensional propensity score matching technique to minimise treatment selection bias, providing key information about risk for women who require these medications for psychiatric stability in pregnancy be at higher risk of gestational diabetes (rate ratio 1.10 ( $95 \% \mathrm{Cl} 0.77$ to 1.57$)$ ), hypertensive disorders of pregnancy (1.12 (0.70 to 1.78)), or venous thromboembolism (0.95 (0.40 to 2.27)). The preterm birth rate, though high among antipsychotic users (14.5\%) and matched non-users (14.3\%), was not relatively different (rate ratio 0.99 (0.78 to 1.26)). Neither birth weight $<3$ rd centile or $>97$ th centile was associated with antipsychotic drug use in pregnancy (rate ratios 1.21 (0.81 to 1.82) and 1.26 (0.69 to 2.29) respectively).

\section{CONCLUSIONS}

Antipsychotic drug use in pregnancy had minimal evident impact on important maternal medical and short term perinatal outcomes. However, the rate of adverse outcomes is high enough to warrant careful assessment of maternal and fetal wellbeing among women prescribed an antipsychotic drug in pregnancy.

\section{Introduction}

There has been a considerable rise in antipsychotic medication use in pregnancy, from 3 per 1000 pregnancies in 2001 to 8 per 1000 in $2007 .^{1}$ Explanations for this include higher conception rates among women with psychotic disorders ${ }^{2}$ and greater use of atypical antipsychotics in the treatment of bipolar and major depressive disorder. ${ }^{3}{ }^{4}$ Decisions about the use of antipsychotic drugs in pregnancy are not straightforward, however. It is recognised that a woman with serious mental illness who discontinues her medication in pregnancy, out concern for fetal harm, may jeopardise her own mental health and her ability to care for her child after delivery. ${ }^{5}$ Yet, there is little information about the use of antipsychotic drugs in pregnancy, especially second generation (“atypical”) antipsychotic drugs.

Case reports, case series, voluntary registries, and small cohort studies suggest that antipsychotic drug exposure in pregnancy is unlikely to cause congenital anomalies but may be associated with preterm birth, low birth weight for gestational age, and neonatal withdrawal effects. ${ }^{6}$ However, most data were based on the older, "typical" antipsychotic drugs, which are now less commonly used. Second generation antipsychotic drugs were first introduced in the early 1990s and differ from earlier agents in terms of their side effects, especially their potentially higher risk of metabolic syndrome and venous thromboembolism. ${ }^{78}$ The few studies that have evaluated atypical antipsychotic drugs in pregnancy were limited in sample size, and in the handling of confounding by indication. ${ }^{9}$ Accordingly, these studies may not have been equipped to evaluate the impact of antipsychotic drug use in pregnancy on important maternal medical and perinatal conditions. 
The objective of the current study was to evaluate common maternal medical conditions that are magnified by pregnancy ${ }^{10}$ and that are also associated with antipsychotic drug use. ${ }^{7}$ We also evaluated important perinatal outcomes in relation to maternal antipsychotic medication use in pregnancy. We used a high dimensional propensity score (HDPS) matched cohort study design to account for observed and unobserved confounding factors that might explain the association between antipsychotic drug use in pregnancy and important maternal and perinatal outcomes.

\section{Methods}

Setting

We conducted a population based cohort study in Ontario, Canada, using health administrative data among women eligible for public drug coverage who had been hospitalised for obstetrical delivery of a live born or stillborn infant between 1 April 2003 and 31 December 2012. These women had universal access to hospital care, physician services, and drug coverage. The study received ethical approval from the research ethics boards at Women's College Hospital (2012-0288-A) and Sunnybrook Health Sciences Centre (ICES project number 20130900343 000) in Toronto, Ontario, Canada.

\section{Data sources}

Multiple linked population health administrative databases housed at the Institute for Clinical Evaluative Sciences (ICES) in Toronto, Ontario, were used for this analysis. Obstetric deliveries were identified using ICES' MOMBABY datafile. This validated datafile comprises all in-hospital deliveries in Ontario, with 98\% successful linkage of maternal and newborn health records. Exposure to antipsychotic drugs and other medications were identified using the Ontario Drug Benefit (ODB) database, which records prescription medication use for those receiving full drug benefits under the provincial drug programme with an error rate of $<1 \%{ }^{11}$ Eligibility for drug coverage includes unemployment, disability, high prescription drug costs relative to net household income, and receipt of home care services. Approximately $70 \%$ of pregnant women with a psychotic disorder receive ODB coverage (source: ICES, unpublished).

Participant demographic information was obtained from the Registered Persons Database (RPDB) that consists of date of birth, sex, postal code, and death date (where applicable). Other data sources were

- Canadian Institutes of Health Information Discharge Abstract Database (CIHI-DAD), to which all hospitals in Ontario submit demographic and clinical information about all hospital admissions and discharges using standard diagnostic ICD-10-CA codes (international classification of diseases, 10th revision, Canadian version) and procedural or interventional CCI codes (Canadian Classification of Health Interventions) $^{12}$

- Ontario Mental Health Reporting System (OMHRS), which contains mental health clinical and adminis- trative data on adult patients from 2005 onward, collected in all facilities in the province of Ontario with designated inpatient mental health beds. ${ }^{1314}$ Before 2005, these data were reported to the CIHI-DAD

- National Ambulatory Care Reporting System (NACRS), which covers emergency department visits, day surgery, and mandated outpatient clinics

- Ontario Health Insurance Plan (OHIP) database, which collects outpatient physician service information from Ontario physicians who are reimbursed after submitting claims to OHIP for each service provided.

Patient level records in all of the above data sources are anonymously linked by a unique identifier number derived from the health card number of every resident of Ontario.

We used validated algorithms from hospital (CIHIDAD) and outpatient fee claims (OHIP database) to identify pre-pregnancy diabetes (sensitivity 0.86 , specificity 0.97$)^{15}$ and chronic hypertension (sensitivity 0.72, specificity 0.95). ${ }^{16}$ Pre-pregnancy medical comorbidity was measured using the John's Hopkins Collapsed Aggregated Diagnosis Group resource utilisation bands (RUB), which classifies individuals into mutually exclusive groups, ranging from those who never use the medical system to high frequency users. ${ }^{17}$ We also used CIHI-DAD and OHIP data to identify pre-pregnancy mental health and addiction service use and diagnoses as well as antenatal care use (including prenatal ultrasonography), ${ }^{1819}$ and the ODB for medications prescribed before and during the index pregnancy.

\section{Participants}

We considered all women who delivered a singleton infant (live birth or stillbirth) in Ontario during the study period. To ensure that all participants were covered under the provincial drug plan during the index pregnancy, we only included those who had filled a provincially funded drug prescription within 180 days before pregnancy and one during pregnancy or within 180 days of delivery. ${ }^{20}$

\section{Exposure groups}

Antipsychotic medication exposure was defined as at least two consecutive prescriptions for an antipsychotic drug filled between the conception date (estimated using the gestational age at birth) and the delivery date. We required that at least one of these prescriptions was filled prior to 27 weeks gestation, corresponding to the first or second trimester of pregnancy. Prescriptions were deemed to be "consecutive" if the second prescription was filled within 1.5 times the number of days' supply of the first prescription. For example, if the first prescription was for 30 days' supply, then the second prescription had to be filled within $1.5 \times 30$ days ( 45 days) thereafter for it to be deemed consecutive. The antipsychotic drugs that were available through the provincial drug plan in the period of study are listed in the supplementary table S1. 
To minimise treatment selection bias, we matched women in the antipsychotic medication group to women not exposed to any antipsychotic drug during pregnancy using a HDPS matching algorithm. ${ }^{21}$ While multivariable modelling and traditional propensity score analyses attempt to address treatment selection bias, they rely on observed confounders. The HDPS algorithm attempts to further minimise residual confounding by indication by also incorporating proxy (or surrogate) variables-healthcare diagnoses, procedures, and drug claims-which, when combined, collectively behave as a good overall proxy for important unobserved confounders. In typical pharmaco-epidemiological studies, HPDS methods result in less biased estimates of treatment effects when benchmarked against those from randomised controlled trials. ${ }^{21}$

In this study, a HDPS algorithm was used to generate propensity scores for all patients in the original cohort. ${ }^{21}$ The data dimensions used were those traditionally available within health claims databases, and included our aforementioned data sources for hospital (CIHIDAD, OMHRS), emergency department (NACRS), and physician billing claim (OHIP) diagnoses and procedures, as well as for prescription drug claims (ODB). Specifically, we included eight data dimensions in the algorithm, as follows: all prescription drug claims $<365$ days before cohort entry (1 dimension, ODB); hospitalisation and emergency department diagnoses and procedures $<365$ days before cohort entry ( 5 dimensions, CIHI-DAD diagnoses and procedures, OMHRS diagnoses, NACRS diagnoses and procedures); and physician service diagnosis and fee codes $<365$ days before cohort entry ( 2 dimensions, OHIP diagnoses and OHIP fee codes). ${ }^{20}$ Within each data dimension the top 200 most prevalent codes, whether diagnostic, procedural, or medication based, were selected, and each code was converted into three binary empirical covariates by rating how often a participant gets a code as "once," "sporadic," or "frequent."

Covariates were selected based on exposure status-covariate relations only, and not covariate outcomes. This method of covariate selection enabled us to efficiently generate a single matched cohort for all outcomes. In addition, exposure-only selection can improve the functionality of the HDPS algorithm when a small number of exposed patients have an outcome event, as was expected in this study. ${ }^{22}$ The relative risk of being in the exposure group was calculated for each of the binary empirical covariates, and the top 500 binary empirical covariates were included in the propensity score estimation. Prescribed antipsychotic medications prior to pregnancy were not considered as covariates, as including them substantially reduced overlap in the propensity score distributions of exposed and unexposed women, making matching difficult. (A full list of exposure covariates included in the HDPS is found in supplementary table S2.)

Each antipsychotic medication user was 1:1 matched with a non-user based on the HDPS score within 0.2 standard deviations and on maternal age at delivery within 3 years.

\section{Study outcomes}

The main maternal medical outcomes were gestational diabetes, the hypertensive disorders of pregnancy (that is, gestational hypertension, pre-eclampsia, eclampsia), and venous thromboembolism, arising within the index pregnancy, the delivery hospitalisation, or within 42 days after delivery (corresponding to the postpartum period). These outcomes, considered to be prevalent, modifiable, and potentially linked to antipsychotic medication exposure, were identified through the CIHIDAD using ICD-10CA codes or in the OHIP database using validated codes. ${ }^{23-25}$

The main perinatal outcomes were preterm birth $<37$ weeks gestation and extremes of newborn weight, namely a birth weight $<3$ rd centile or $>97$ th centile for same sex and same gestational age. ${ }^{26}$ These outcomes were selected because they are prevalent and are major determinants of perinatal morbidity and mortality. ${ }^{27-30}$ Perinatal outcomes were based on validated ICD-10CA diagnoses during the index birth hospitalisation (CIHIDAD). ${ }^{2431}$

Secondary outcomes included a number of other key adverse maternal and perinatal outcomes that were derived using the same databases as for the primary outcomes. ${ }^{32}$ These included specific maternal and neonatal conditions, health service utilisation, and mortality. A list of the data sources and codes used to define each outcome is found in supplementary table S1.

\section{Statistical analyses}

We reported the baseline characteristics of antipsychotic users and non-users prior to matching (that is, the "unmatched cohort"), and of antipsychotic users and non-users after matching on HDPS score (the "matched cohort"). For the unmatched cohort, Poisson regression analysis was used to generate relative risks and 95\% confidence intervals, comparing the risk of each study outcome among antipsychotic users relative to non-users.

For the matched cohort, conditional Poisson regression analysis was used to compare the risk of each study outcome among antipsychotic users relative to their matched non-users. We further adjusted for a prescribed selective serotonin reuptake inhibitor (SSRI), non-SSRI, mood stabiliser, or benzodiazepine medication during the index pregnancy because standardised differences exceeded 0.10 on these key prognostic variables even after matching.

To provide more details about the association between specific antipsychotic medications and the main outcomes, we further restricted the analyses to the atypical antipsychotics, as well as exclusively prescribed quetiapine, olanzapine, and risperidone. We conducted sensitivity analyses for the main outcomes according to the pregnancy trimester of medication exposure. For selected secondary outcomes likely to be associated with time-specific exposures (that is, first trimester drug exposure and congenital malformations, or third trimester drug exposure and neonatal adaptation syndrome), we conducted sensitivity analyses restricting exposure to the appropriate time periods. 
We also conducted sensitivity analyses for the main maternal medical and main perinatal outcomes, and for the secondary outcome of congenital malformations, in which we excluded those matched pairs where a nonuser was prescribed an antipsychotic medication between one and 60 days before the estimated date of conception.

All analyses were conducted using SAS statistical software, version 9.3 of the SAS System for Unix.

Patient involvement-There was no direct patient involvement in this study. ICES datasets comprise linked de-identified health administrative information.

\section{Results}

There were 52615 singleton live births or stillbirths to women eligible for public drug coverage during the study period. Of these, we identified 1913 pregnancies during which an antipsychotic drug was prescribed, of which 1323 pregnancies (in 1209 unique women) had two consecutive prescriptions, one of which was filled in the first or second trimester. We identified 50702 pregnancies (to 40314 unique women) with no antipsychotic prescriptions. Within both groups, we randomly selected one birth for each woman, such that no woman contributed more than one birth to the analysis.

Out of 1209 antipsychotic users in the overall cohort, 1021 (84\%) were successfully matched using the HDPS algorithm (table 1). About $90 \%$ of matched antipsychotic users were exclusively prescribed an atypical antipsychotic medication, and, of these, 556 were exclusively prescribed quetiapine, 166 olanzapine, and 112 risperidone.

Unmatched antipsychotic users were older and had higher baseline medical morbidity than unmatched non-users (table 1). They also had more prenatal visits and were less likely to receive antenatal care from an obstetrician. There were substantial differences between unmatched users and non-users for all mental health measures. After matching, there was marked attenuation in the standardised differences for all

Table 1 | Characteristics of 41523 women who were categorised by whether they were prescribed an antipsychotic drug during pregnancy. Of these, 1021 antipsychotic drug users were matched 1:1 with 1021 non-users by means of a high dimensional propensity score (HDPS) algorithm. Values are numbers (percentages) unless stated otherwise

\begin{tabular}{|c|c|c|c|c|c|c|}
\hline \multirow[b]{2}{*}{ Measure } & \multicolumn{3}{|c|}{ Unmatched cohort } & \multicolumn{3}{|l|}{ Matched cohort } \\
\hline & $\begin{array}{l}\text { Antipsychotic } \\
\text { users }(n=1209)\end{array}$ & $\begin{array}{l}\text { Non-users } \\
(n=40314)\end{array}$ & $\begin{array}{l}\text { Standardised } \\
\text { difference }\end{array}$ & $\begin{array}{l}\text { Antipsychotic } \\
\text { users }(n=1021)\end{array}$ & $\begin{array}{l}\text { Non-users } \\
(n=1021)\end{array}$ & $\begin{array}{l}\text { Standardised } \\
\text { difference }\end{array}$ \\
\hline \multicolumn{7}{|l|}{ Demographic characteristics } \\
\hline Mean (SD) age (years) & $28.8(6.1)$ & $26.7(6.3)$ & 0.35 & $28.8(6.2)$ & $28.8(6.2)$ & $<0.01$ \\
\hline Median (interquartile range) parity & $1(0-2)$ & $1(0-2)$ & 0.14 & $1(0-2)$ & $1(0-2)$ & 0.07 \\
\hline Lowest neighbourhood income quintile & $613(50.7)$ & $20722(51.4)$ & 0.01 & $501(49.1)$ & $458(45.0)$ & 0.08 \\
\hline Urban residence & $1106(91.5)$ & $36452(90.4)$ & 0.04 & $930(91.1)$ & $909(89.2)$ & 0.06 \\
\hline \multicolumn{7}{|l|}{ Medical morbidity } \\
\hline Pre-pregnancy diabetes & $52(4.3)$ & $1303(3.2)$ & 0.06 & $39(3.8)$ & $42(4.1)$ & 0.02 \\
\hline Pre-pregnancy chronic hypertension & $44(3.6)$ & $1276(3.2)$ & 0.03 & $39(3.8)$ & $49(4.8)$ & 0.05 \\
\hline Venous thromboembolism <1 year before index pregnancy & $28(2.3)$ & $672(1.7)$ & 0.05 & $25(2.5)$ & $23(2.3)$ & 0.01 \\
\hline High resource utilisation band of 4 or 5 & $927(76.7)$ & $29050(72.1)$ & 0.42 & $767(73.1)$ & $799(78.3)$ & 0.11 \\
\hline \multicolumn{7}{|l|}{ Prenatal care } \\
\hline Prenatal ultrasound before 20 weeks gestation & $1049(86.8)$ & $35372(87.8)$ & 0.03 & $885(86.7)$ & $884(86.6)$ & $<0.01$ \\
\hline Mean (SD) No of prenatal visits & $18.3(10.3)$ & $15.3(7.2)$ & 0.33 & $18.1(10.2)$ & $18.5(11.0)$ & 0.03 \\
\hline Prenatal care provider: & & & 0.15 & & & 0.06 \\
\hline Primary care & $288(23.8)$ & $8002(19.9)$ & & $244(23.9)$ & $230(22.5)$ & \\
\hline Obstetrician & $276(22.8)$ & $11362(28.2)$ & & $234(22.9)$ & $237(23.2)$ & \\
\hline Primary care and obstetrician & $614(50.8)$ & $19556(48.5)$ & & $514(50.3)$ & $516(50.5)$ & \\
\hline Other (such as midwife) & $31(2.6)$ & $1394(3.5)$ & & $29(2.8)$ & $38(3.7)$ & \\
\hline \multicolumn{7}{|l|}{ Mental health measures } \\
\hline Mean (SD) No of outpatient visits $<1$ year before index pregnancy & $8.46(11.1)$ & $1.48(5.3)$ & 0.80 & $7.83(10.8)$ & $9.15(15.0)$ & 0.10 \\
\hline Emergency department visit <1 year before index pregnancy & $326(27.0)$ & $2211(5.5)$ & 0.61 & $242(23.7)$ & $228(22.3)$ & 0.03 \\
\hline Admission $<1$ year before index pregnancy & $249(20.6)$ & $552(1.4)$ & 0.65 & $158(15.5)$ & $133(13.0)$ & 0.07 \\
\hline Prescribed antipsychotic drug $<1$ year before index pregnancy* & $1075(88.9)$ & $1334(3.3)$ & 3.35 & $895(87.7)$ & $274(26.8)$ & 1.56 \\
\hline \multicolumn{7}{|l|}{ Psychiatric diagnoses before index pregnancy: } \\
\hline Psychotic disorder & $429(35.5)$ & $675(1.69)$ & 0.97 & $319(31.2)$ & $160(15.7)$ & 0.37 \\
\hline Bipolar disorder or major depression & $937(77.5)$ & $8708(21.6)$ & 1.35 & $758(74.2)$ & $673(65.9)$ & 0.18 \\
\hline Alcohol or substance disorder (including smoking) & $569(47.1)$ & $5523(13.7)$ & 0.78 & $458(44.9)$ & $415(40.7)$ & 0.08 \\
\hline Personality disorder & $393(32.5)$ & $1816(4.5)$ & 0.77 & 295 (28.9) & $231(22.6)$ & 0.14 \\
\hline \multicolumn{7}{|l|}{ Non-antipsychotic drug prescribed in the pregnancy: } \\
\hline SSRI & $364(30.1)$ & $1981(4.9)$ & 0.70 & $303(29.7)$ & $204(20.0)$ & 0.23 \\
\hline Non-SSRI & $322(26.6)$ & $989(2.5)$ & 0.73 & $264(25.9)$ & $149(14.6)$ & 0.28 \\
\hline Mood stabiliser & $167(13.8)$ & $331(0.8)$ & 0.52 & $105(10.3)$ & $62(6.1)$ & 0.15 \\
\hline Benzodiazepine & $306(25.3)$ & $630(1.6)$ & 0.74 & $222(21.7)$ & $135(13.2)$ & 0.23 \\
\hline
\end{tabular}


baseline characteristics. Although reduced by the matching process, notable standardised differences persisted between matched users and non-users for psychotic and major mood disorders, as well as for prescribed antidepressant drugs, mood stabilisers, and benzodiazepines (table 1). Nearly $88 \%$ of antipsychotic users, compared with $27 \%$ of matched non-users, were prescribed an antipsychotic medication within one year before the estimated date of conception.

\section{Main outcomes}

For the main maternal outcomes assessed within the unmatched cohort, event rates were higher among antipsychotic users than non-users for gestational diabetes $(7.7 \% v 6.2 \%)$ and the hypertensive disorders of pregnancy (5.2\% v 3.5\%), but not for venous thromboembolism (table 2). In the matched cohort, these differences were no longer significant, before or after further adjustment for other prescribed psychotropic drugs (table 2). On restricting our analysis to atypical antipsychotics and specific drugs, the results were unchanged (fig 1).

For the main perinatal outcomes assessed within the unmatched cohort, event rates were higher among antipsychotic users than non-users for preterm birth (14.8\% $v 10.3 \%)$ and birth weight $>97$ th centile (3.7\% v 2.6\%) (table 2). After matching, none of these outcomes was significantly different between antipsychotic users and non-users (table 2), even when restricting our analysis to atypical antipsychotics and specific drugs (fig 2).

\section{Secondary outcomes}

In the unmatched cohort, women using antipsychotics had significantly higher rates of placental abruption, labour induction, caesarean section, and operative vaginal delivery, as well as readmission to hospital (table 3). Likewise, their infants were at significantly higher risk of preterm birth <32 weeks (relative risk 1.61 (95\% CI 1.19 to 2.16)), intraventricular haemorrhage (2.84 (1.53 to 5.27)), seizures (4.30 (2.22 to 8.33)), sepsis (2.26 (1.53 to 3.32)), and especially, neonatal adaptation syndrome (7.06 (5.91 to 8.45)) (table 3). However, in the matched cohort, only labour induction (adjusted relative risk 1.23 (1.03 to 1.48)) and operative vaginal delivery (1.55 (1.10 to 2.19)) were associated with antipsychotic drug use in pregnancy (table 3).

\section{Sensitivity analyses}

Assessment of the main maternal and perinatal outcomes by trimester of exposure in the matched cohort was generally consistent with the primary analyses (supplementary table S3). Birth weight $>97$ th centile was more common among those prescribed an antipsychotic agent in the second or third trimester of pregnancy compared with matched controls, but the associations were no longer significant after adjusting for other prescribed non-antipsychotic psychotropic medications. Restricting antipsychotic exposure to the first trimester of pregnancy in the assessment of congenital malformations, and to the third trimester for consideration of the neonatal adaptation syndrome, no meaningful changes in the main results were observed (supplementary table S4). For the matched analyses, 75 women in the non-user group were prescribed an antipsychotic medication in the 60 days before the conception date. Excluding these 75 matched pairs did not appreciably change the adjusted relative risks for the main maternal and

\begin{tabular}{|c|c|c|c|c|c|}
\hline \multirow[b]{2}{*}{ Outcome and use of antipsychotic medication } & \multicolumn{2}{|c|}{ Unmatched cohort } & \multicolumn{3}{|c|}{ Matched cohort } \\
\hline & $\begin{array}{l}\text { No (\%) with } \\
\text { outcome }\end{array}$ & $\begin{array}{l}\text { Relative risk } \\
(95 \% \mathrm{Cl})\end{array}$ & $\begin{array}{l}\text { No (\%) with } \\
\text { outcome }\end{array}$ & $\begin{array}{l}\text { Relative risk } \\
(95 \% \mathrm{Cl})\end{array}$ & $\begin{array}{l}\text { Adjusted relative } \\
\text { risk }(95 \% \mathrm{Cl})^{*}\end{array}$ \\
\hline \multicolumn{6}{|l|}{ Main maternal medical outcomes } \\
\hline \multicolumn{6}{|l|}{ Gestational diabetes: } \\
\hline Non-users & $2497(6.2)$ & 1.00 (referent) & $62(6.1)$ & 1.00 (referent) & 1.00 (referent) \\
\hline Antipsychotic users & $93(7.7)$ & 1.24 (1.01 to 1.53$)$ & $71(7.0)$ & $1.15(0.82$ to 1.61$)$ & 1.10 (0.77 to 1.57$)$ \\
\hline \multicolumn{6}{|l|}{ Hypertensive disorders of pregnancy: } \\
\hline Non-users & $1409(3.5)$ & 1.00 (referent) & $42(4.1)$ & 1.00 (referent) & 1.00 (referent) \\
\hline Antipsychotic users & $63(5.2)$ & 1.49 (1.16 to 1.92$)$ & $48(4.7)$ & 1.14 (0.76 to 1.73$)$ & $1.12(0.70$ to 1.78$)$ \\
\hline \multicolumn{6}{|l|}{ Venous thromboembolism: } \\
\hline Non-users & $4264(1.1)$ & 1.00 (referent) & $13(1.3)$ & 1.00 (referent) & 1.00 (referent) \\
\hline Antipsychotic users & $12(1.0)$ & 0.95 (0.53 to 1.68$)$ & $12(1.2)$ & 0.92 (0.42 to 2.02$)$ & $0.95(0.40$ to 2.27$)$ \\
\hline \multicolumn{6}{|l|}{ Main perinatal outcomes } \\
\hline \multicolumn{6}{|l|}{ Preterm birth (<37 weeks gestation): } \\
\hline Non-users & $4158(10.3)$ & 1.00 (referent) & $146(14.3)$ & 1.00 (referent) & 1.00 (referent) \\
\hline Antipsychotic users & $179(14.8)$ & 1.51 (1.29 to 1.78$)$ & $148(14.5)$ & $1.01(0.81$ to 1.27$)$ & $0.99(0.78$ to 1.26$)$ \\
\hline \multicolumn{6}{|l|}{ Small for gestational age (birth weight $<3$ rd centile): } \\
\hline Non-users & $2081(5.2)$ & 1.00 (referent) & $51(5.1)$ & 1.00 (referent) & 1.00 (referent) \\
\hline Antipsychotic users & $74(6.2)$ & 1.20 (0.95 to 1.53$)$ & $62(6.1)$ & $1.22(0.84$ to 1.77$)$ & 1.21 (0.81 to 1.82$)$ \\
\hline \multicolumn{6}{|l|}{ Large for gestational age (birth weight $>97$ th centile): } \\
\hline Non-users & $1029(2.6)$ & 1.00 (referent) & $23(2.3)$ & 1.00 (referent) & 1.00 (referent) \\
\hline Antipsychotic users & $44(3.7)$ & $1.44(1.06$ to 1.96$)$ & $36(3.6)$ & 1.64 (0.96 to 2.78$)$ & 1.26 (0.69 to 2.29$)$ \\
\hline
\end{tabular}

*Adjusted for a prescribed selective serotonin reuptake inhibitor (SSRI), non-SSRI, mood stabiliser, or benzodiazepine during the index pregnancy. 


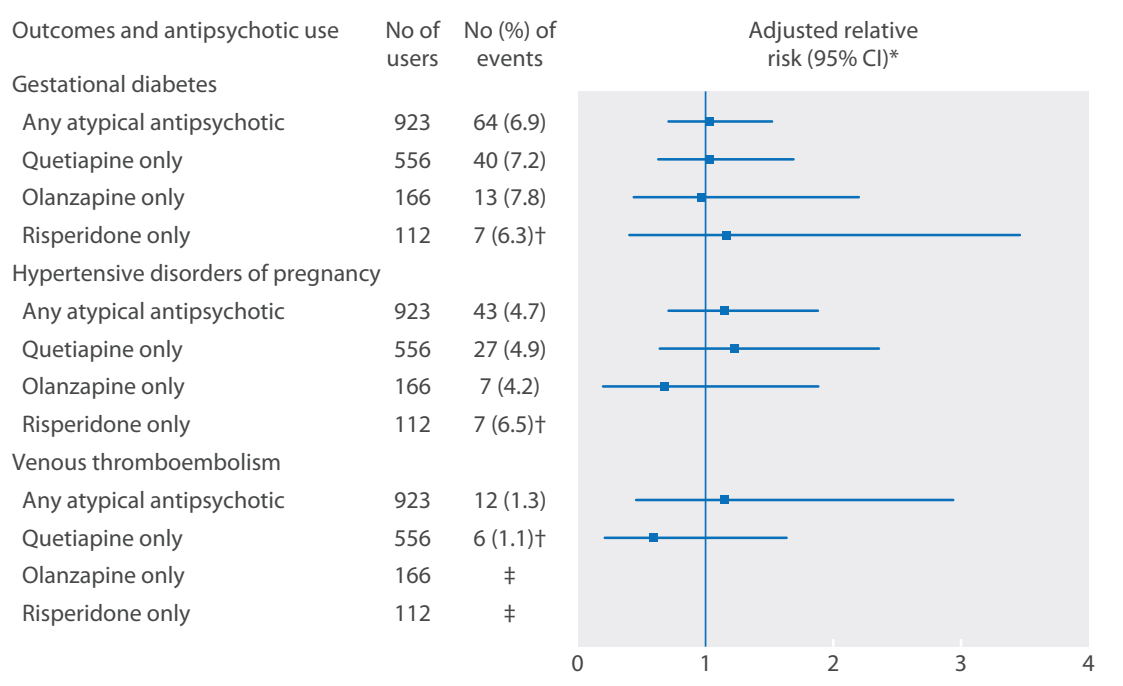

* Adjusted for a prescribed selective serotonin reuptake inhibitor (SSRI), non-SSRI, mood stabiliser, and/or benzodiazepine medication during the index pregnancy

† Crude relative risks presented because so few events

‡ Event rates and/or relative risks are too low to report

Fig 1 Main maternal medical outcomes in a cohort of 1021 women who were prescribed an antipsychotic drug during pregnancy and who were matched 1:1 with 1021 non-users by means of a high dimensional propensity score (HDPS) algorithm. Relative risks compare antipsychotic drug users-restricted to use of any atypical antipsychotic drug, and specifically, quetiapine, olanzapine, or risperidone-with matched non-users

perinatal outcomes or for the outcome of any congenital malformation (supplementary table S5).

\section{Discussion}

Principal findings

Antipsychotic drug use in pregnancy did not substantially worsen maternal medical or short term perinatal outcomes in a cohort of women closely matched on baseline characteristics using a HDPS algorithm. The

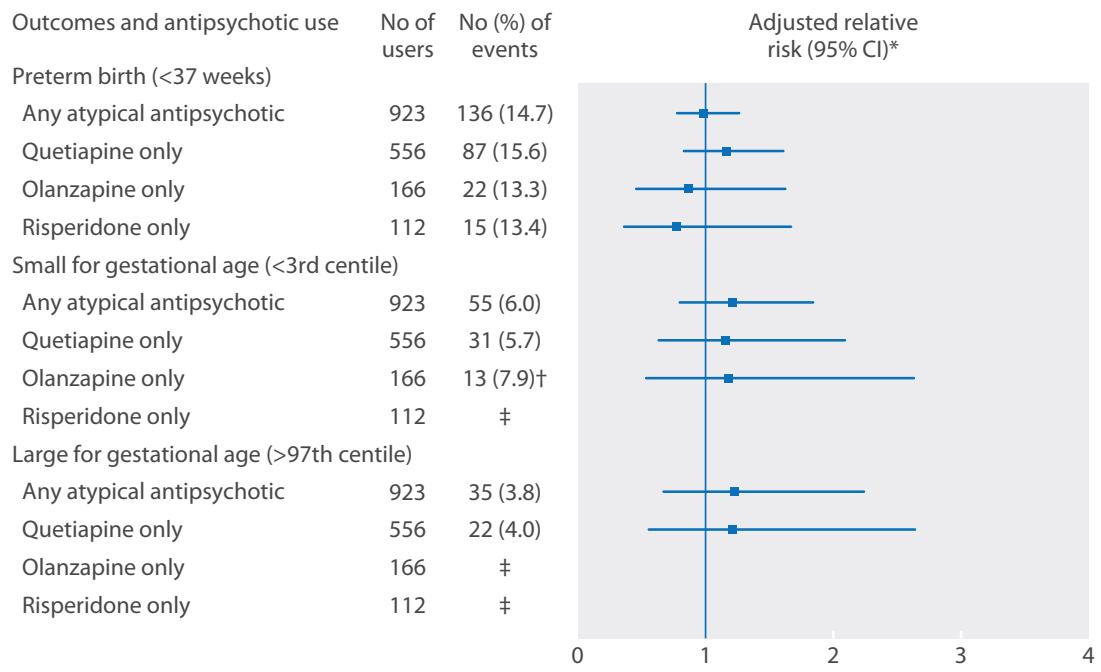

* Adjusted for a prescribed selective serotonin reuptake inhibitor (SSRI), non-SSRI, mood stabiliser, and/or benzodiazepine medication during the index pregnancy

† Crude relative risks presented because so few events

‡ Event rates and/or relative risks are too low to report

Fig 2 Main perinatal outcomes in a cohort of 1021 women who were prescribed an antipsychotic drug during pregnancy and who were matched 1:1 with 1021 non-users by means of a high dimensional propensity score (HDPS) algorithm. Relative risks compare antipsychotic drug users-restricted to use of any atypical antipsychotic drug, and specifically, quetiapine, olanzapine, or risperidone-with matched non-users only exceptions were a slightly higher risk of labour induction and vaginal delivery among exposed pregnancies. However, antipsychotic users had outcome event rates that were much higher than in the general population..$^{33}$ This was true for the main maternal outcomes of the hypertensive disorders of pregnancy and venous thromboembolism, and for the main perinatal outcomes, including almost a $14 \%$ rate of preterm birth, about twice that in the general population. ${ }^{33}$ Although there were few events, the observed neonatal mortality rate of $1 \%$ was twice that of the general population. ${ }^{33}$

\section{Strengths and limitations of study}

We included a large, population based cohort of women, using a clear definition of the study exposure followed by meaningful outcomes. In contrast to other studies, we used an HDPS matching procedure to create a comparison group closely matched on measured baseline characteristics. This method also attempts to balance individuals on a number of other factors that may be proxies for confounders not easily identified in our databases, thus reducing the potential impact of unmeasured confounding. By presenting effect estimates for an unmatched cohort, as well as a matched cohort, we were able to show the potential magnitude of bias created from confounding by indication.

We included only women for whom information on prescription medications was available through a provincially covered drug plan. Individuals eligible for this drug plan tend to have worse health states, greater disability, and are of lower socioeconomic status compared with individuals who pay privately for their medication. Therefore, these results may not be entirely generalisable to antipsychotic users who are healthier and of higher socioeconomic status. Nonetheless, the women included herein warrant careful study of the impact of medication use in pregnancy, as they may be more susceptible to adverse outcomes than healthier women.

The use of HDPS matching is meant to minimise residual confounding and to approximate relative risk estimates observed in a randomised clinical trial. Nonetheless, we did not achieve a balanced match on some characteristics. Most notably, the groups differed after matching on additionally prescribed non-antipsychotic psychotropic medication. Because other psychotropic drugs could affect maternal and perinatal outcomes, ${ }^{34-37}$ they were adjusted for, however. It is reassuring that, on doing so, the results for the outcomes in the main analyses did not change. Nonetheless, residual confounding may have persisted due to inadequate capture of variables such as smoking and obesity or due to unmeasured factors such as psychiatric symptoms. ${ }^{3839}$

We allowed for a broad time exposure window for antipsychotic medication use. This would be expected to reduce the accurate capture of the timing of medication exposure in pregnancy, and thus, the specific determination of time sensitive outcomes. For this reason, we conducted sensitivity analyses for the main outcomes by trimester of exposure, including limiting 
Table 3 | Secondary maternal and perinatal outcomes in a cohort of 41523 women who were categorised by whether they were prescribed an antipsychotic drug during pregnancy ( $n=1209)$ or not ( $n=40314)$. Of these, 1021 antipsychotic drug users were matched 1:1 with 1021 non-users by means of a high dimensional propensity score (HDPS) algorithm

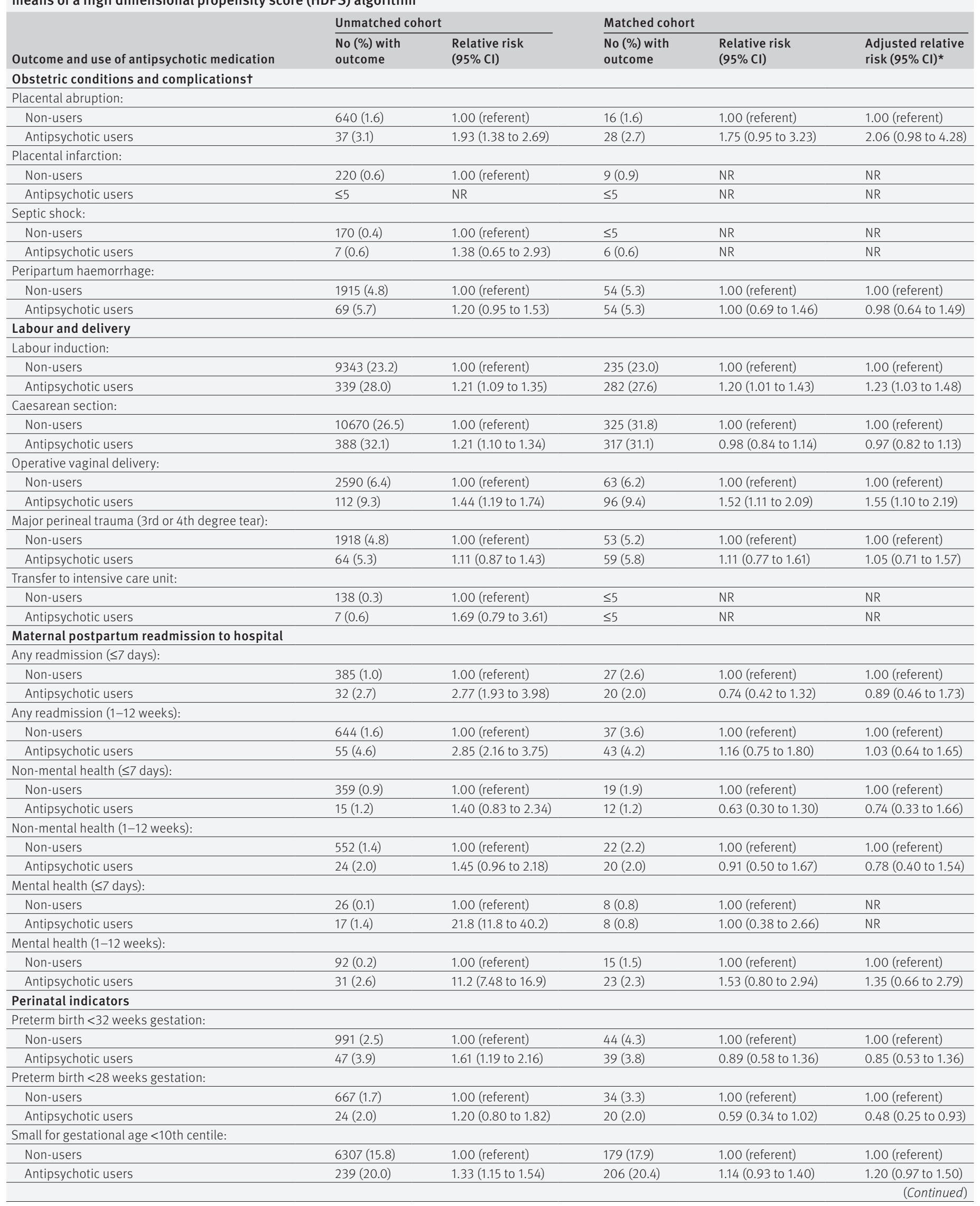




\begin{tabular}{|c|c|c|c|c|c|}
\hline \multirow[b]{2}{*}{ Outcome and use of antipsychotic medication } & \multicolumn{2}{|c|}{ Unmatched cohort } & \multicolumn{3}{|c|}{ Matched cohort } \\
\hline & $\begin{array}{l}\text { No (\%) with } \\
\text { outcome }\end{array}$ & $\begin{array}{l}\text { Relative risk } \\
(95 \% \mathrm{Cl})\end{array}$ & $\begin{array}{l}\text { No (\%) with } \\
\text { outcome }\end{array}$ & $\begin{array}{l}\text { Relative risk } \\
(95 \% \mathrm{Cl})\end{array}$ & $\begin{array}{l}\text { Adjusted relative } \\
\text { risk }(95 \% \mathrm{Cl})^{\star}\end{array}$ \\
\hline \multicolumn{6}{|l|}{ Large for gestational age $>90$ th centile: } \\
\hline Non-users & $3091(7.8)$ & 1.00 (referent) & $77(7.7)$ & 1.00 (referent) & 1.00 (referent) \\
\hline Antipsychotic users & $108(9.0)$ & $1.18(0.97$ to 1.45$)$ & $88(8.7)$ & 1.15 (0.84 to 1.56$)$ & 1.07 (0.76 to 1.51$)$ \\
\hline \multicolumn{6}{|l|}{ Neonatal complications } \\
\hline \multicolumn{6}{|l|}{ Respiratory distress syndrome: } \\
\hline Non-users & $597(1.5)$ & 1.00 (referent) & $30(2.9)$ & 1.00 (referent) & 1.00 (referent) \\
\hline Antipsychotic users & $33(2.7)$ & 1.87 (1.31 to 2.66$)$ & $26(2.6)$ & $0.87(0.51$ to 1.47$)$ & $0.82(0.46$ to 1.43$)$ \\
\hline \multicolumn{6}{|l|}{ Seizure: } \\
\hline Non-users & $78(0.2)$ & 1.00 (referent) & $7(0.7)$ & 1.00 (referent) & NR \\
\hline Antipsychotic users & $10(0.8)$ & $4.30(2.22$ to 8.33$)$ & $9(0.9)$ & $1.29(0.48$ to 3.45$)$ & NR \\
\hline \multicolumn{6}{|l|}{ Sepsis: } \\
\hline Non-users & $419(1.0)$ & 1.00 (referent) & $14(1.4)$ & 1.00 (referent) & 1.00 (Referent) \\
\hline Antipsychotic users & $28(2.2)$ & 2.26 (1.53 to 3.32$)$ & $24(2.4)$ & 1.71 (0.89 to 3.31$)$ & $1.80(0.84$ to 3.85$)$ \\
\hline \multicolumn{6}{|l|}{ Intraventricular haemorrhage: } \\
\hline Non-users & $130(0.3)$ & 1.00 (referent) & $6(0.6)$ & 1.00 (referent) & NR \\
\hline Antipsychotic users & $11(0.9)$ & $2.84(1.53$ to 5.27$)$ & $8(0.8)$ & 1.33 (0.46 to 3.84$)$ & NR \\
\hline \multicolumn{6}{|l|}{ Persistent fetal circulation: } \\
\hline Non-users & $74(0.2)$ & NR & $\leq 5$ & NR & NR \\
\hline Antipsychotic users & $\leq 5$ & NR & $\leq 5$ & NR & NR \\
\hline \multicolumn{6}{|l|}{ Neonatal adaptation syndrome: } \\
\hline Non-users & $858(2.1)$ & 1.00 (referent) & $111(10.9)$ & 1.00 (referent) & 1.00 (referent) \\
\hline Antipsychotic users & $161(13.3)$ & 7.06 (5.91 to 8.45$)$ & $132(12.9)$ & 1.19 (0.92 to 1.53$)$ & 1.15 (0.88 to 1.50$)$ \\
\hline \multicolumn{6}{|l|}{ Congenital or neonatal infection: } \\
\hline Non-users & $145(0.4)$ & 1.00 (referent) & $10(1.0)$ & 1.00 (referent) & NR \\
\hline Antipsychotic users & $7(0.6)$ & $1.61(0.75$ to 3.45$)$ & $7(0.7)$ & $0.70(0.27$ to 1.84$)$ & NR \\
\hline \multicolumn{6}{|l|}{ Congenital malformations: } \\
\hline Non-users & $1589(4.0)$ & 1.00 (referent) & $40(4.0)$ & 1.00 (referent) & 1.00 (referent) \\
\hline Antipsychotic users & $50(4.2)$ & 1.05 (0.79 to 1.41$)$ & $44(4.4)$ & $1.10(0.72$ to 1.69$)$ & 1.19 (0.75 to 1.91) \\
\hline \multicolumn{6}{|l|}{ Maternal and perinatal mortality } \\
\hline \multicolumn{6}{|l|}{ Stillbirth > 20 weeks gestation: } \\
\hline Non-users & $349(09)$ & 1.00 (referent) & $16(1.6)$ & 1.00 (referent) & NR \\
\hline Antipsychotic users & $12(1.0)$ & 1.15 (0.64 to 2.05$)$ & $9(0.9)$ & $0.56(0.25$ to 1.27$)$ & NR \\
\hline \multicolumn{6}{|l|}{ Neonatal mortality $<90$ days: } \\
\hline Non-users & $184(0.5)$ & 1.00 (referent) & $6(0.6)$ & 1.00 (referent) & NR \\
\hline Antipsychotic users & $9(0.7)$ & 1.64 (0.84 to 3.20$)$ & $9(0.9)$ & $1.50(0.53$ to 4.21$)$ & NR \\
\hline \multicolumn{6}{|l|}{ Maternal mortality 0-90 days postpartum: } \\
\hline Non-users & $9(0.0)$ & NR & $0(0.0)$ & NR & NR \\
\hline Antipsychotic users & $\leq 5$ & NR & $\leq 5$ & NR & NR \\
\hline
\end{tabular}

congenital malformations to exposure in the first trimester and the neonatal adaptation syndrome to exposure in the third trimester.

We did not specifically assess for tardive dyskinesia or other movement disorders in the mother, nor we did not assess the effects of antipsychotic drugs on maternal or infant cardiac conduction, such as QT interval prolongation. While we did not have access to clinical data with that level of detail, we did assess for rare adverse neonatal cardiac and neurological outcomes, such as cardiac arrest and status epilepticus.

Our subgroup analyses were based on relatively large samples of 923 atypical antipsychotic users and 556 quetiapine users. However, there were only 166 exclusive olanzapine users and 112 exclusive risperidone users, producing relatively underpowered subgroups analyses for most rare outcomes.

\section{Context in relation to existing literature}

Two large population based studies previously evaluated the use of antipsychotic medications in pregnancy. Reis and Kallen evaluated 570 Swedish women with self reported use of antipsychotic medications in early pregnancy and compared them with unexposed women on a wide range of outcomes. ${ }^{40}$ In contrast to our study, they observed an increased risk of gestational diabetes (adjusted odds ratio 1.78 (95\% CI 1.04 to 3.71)) and caesarean delivery (1.43 (1.14 to 1.74)), but their analyses only accounted for confounding by year of birth, maternal age, parity, smoking, and prior miscarriage. 
Importantly, their findings are consistent with those generated for our unmatched cohort.

A second Swedish population based study, using a prescription drug database, compared 507 pregnancies with antenatal exposure to an antipsychotic medication with 357696 unexposed pregnancies with respect to the risk for gestational diabetes, small and large birth weight for gestational age, and macrocephaly by trimester of exposure. ${ }^{41}$ After controlling for maternal body mass index, maternal age, country of origin, cohabitation, and smoking-but not use of other psychotropic medications-there was no significant difference in most outcomes, which is generally consistent with our findings. However, they observed a higher risk of macrocephaly among infants exposed to olanzapine and clozapine (adjusted odds ratio 3.02 (95\% CI 1.60 to 5.71)), an outcome that we did not consider. These two cohort studies ${ }^{4041}$ and others ${ }^{4142}$ have advanced our understanding about the safety of antipsychotic medication use in pregnancy, but none dealt sufficiently with evident confounding by indication by using HDPS, as we did.

\section{Explanation of current findings}

Our findings suggest that antipsychotic medication use in pregnancy poses a minimal risk for adverse outcomes for the pregnant woman and her fetus, in and of itself. However, it is of great interest that the rates of adverse perinatal outcomes observed in our matched cohort of antipsychotic users were higher than in the unmatched controls or in the general population. ${ }^{33}$ This means that women and fetuses exposed to antipsychotic drugs seem to be at higher absolute risk of adverse outcomes, even though they approximated the risk of matched non-users with similarly high pre-pregnancy morbidity. In the matched cohort, about $88 \%$ of antipsychotic users and $27 \%$ of non-users had been prescribed an antipsychotic medication within the year before conception. As such, it remains possible that atypical antipsychotic drugs worsened a woman's metabolic function before pregnancy. This is supported by the fact that the baseline rates of diabetes (4\%) and chronic hypertension (4\%) were higher for women in our cohort than in the general population, whose respective rates are $1 \%$ and $1.7 \% .{ }^{42} 43$ We do not know the reason for the observed higher risk of labour induction and operative vaginal delivery. Given that these were the only statistically significant outcomes among a long list of secondary outcomes, it simply could be the play of chance, or because their caregivers were more inclined to set the timing or mode of delivery in the face of a major mental illness.

It is important to address our findings about neonatal adaptation syndrome, one of our secondary outcomes. In 2011 the US Federal Drug Administration issued a warning about risk for extrapyramidal symptoms or withdrawal in the newborn after exposure to antipsychotic medication in utero (www.fda.gov/Drugs/DrugSafety/ucm243903.htm). This warning was based on 69 case reports, most of which were confounded by other potential aetiological factors. In our study, the sevenfold increased risk for neonatal adaptation syndrome in the unmatched cohort was reduced to a small, non-significant relative risk in the matched cohort analysis. This suggests that neonatal adaptation syndrome following antenatal antipsychotic exposure could be attributable to other factors, such as increased rates of concomitant medication use, substance use, or alcohol use in women prescribed an antipsychotic drug rather than due to the medication itself. Certainly, this is an area in need of detailed study about drug initiation and cessation before delivery, and the use of a standardised measure of neonatal adaptation syndrome.

\section{Conclusions and future directions}

Decisions about medication use in pregnancy involve weighing the indications and potential benefits over that of potential harm to mother and child. Women requiring antipsychotic medications are at higher absolute risk for certain adverse maternal and perinatal outcomes compared with the general population. As such, it is prudent to closely monitor the medical health of these women before and during, pregnancy, with particular attention to issues related to diabetes, hypertension, preterm birth, and fetal growth. However, antipsychotic medications themselves do not seem to have an extensive negative impact on important measures of maternal medical and short term perinatal wellbeing. This is reassuring, at least in the short term, for a woman with serious mental illness who requires an antipsychotic medication to achieve and maintain psychiatric stability during and after pregnancy.

Contributors: SNV, TG, VHT, and JGR conceived of the study. All authors performed data analysis and interpretation. SNV drafted the manuscript; SNV, TG, ASW, VHT, and JGR revised the manuscript; and all authors approval the final version. SNV is guarantor for the study.

Funding: This study was supported by a grant from the Canadian Institutes of Health Research (CIHR). It was also supported by the Institute for Clinical Evaluative Sciences (ICES), which is funded by an annual grant from the Ontario Ministry of Health and Long-Term Care (MOHLTC). The opinions, results, and conclusions reported in this paper are those of the authors and are independent from the funding sources. No endorsement by ICES or the Ontario MOHLTC is intended or should be inferred.

Role of the study sponsors: The study sponsors provided the operating costs and infrastructure to support the research. No funding bodies had any role in the study design, data collection, analysis, decision to publish, or preparation of the manuscript.

Competing interests: All authors have completed the ICMJE uniform disclosure form at www.icmje.org/coi_disclosure.pdf and declare that no authors (nor their institutions) received payments for their efforts on this project. Unrelated to this project, SNV has received a one-time consulting fee from Multi-Dimensional Health Care (MDH) consulting for the development of continuing healthcare activities related to perinatal mental health; VHT receives funding from Bristol-Myers Squibb for an investigator initiated study and has been a speaker for Astra-Zeneca, Bristol-Myers Squibb, Eli Lilly, and Lundbeck. The remaining authors declare no competing interests: no support from any organisation for the submitted work; no financial relationships with any organisations that might have an interest in the submitted work in the previous three years, no other relationships or activities that could appear to have influenced the submitted work.

Transparency: SNV affirms that the manuscript is an honest, accurate, and transparent account of the study being reported; that no important aspects of the study have been omitted; and that any discrepancies from the study as planned have been explained. All authors, external and internal, had full access to all of the data (including statistical reports and tables) in the study and can take responsibility for the integrity of the data and the accuracy of the data analysis. 
Data sharing: ICES is named as a prescribed entity under Section 45(1) of Ontario's Personal Health Information Protection Act, 2004 (PHIPA). As a requirement of having this status in PHIPA, ICES policies, practices and procedures are reviewed and approved by the Ontario Information and Privacy Commissioner. Access to raw data is governed by confidentiality agreements between ICES and independent investigators as per PHIPHA guidelines.

This is an Open Access article distributed in accordance with the Creative Commons Attribution Non Commercial (CC BY-NC 4.0) license, which permits others to distribute, remix, adapt, build upon this work non-commercially, and license their derivative works on different terms, provided the original work is properly cited and the use is non-commercial. See: http://creativecommons.org/licenses/ by-nc/4.0/.

1 Toh S, Li Q, Cheetham TC, et al. Prevalence and trends in the use of antipsychotic medications during pregnancy in the US, 2001-2007: a population-based study of 585,615 deliveries. Arch Womens Ment Health 2013;16:149-57.

2 Vigod SN, Seeman MV, Ray JG, et al. Temporal trends in general and age-specific fertility rates among women with schizophrenia (1996-2009): a population-based study in Ontario, Canada. Schizophr Res 2012;139:169-75.

3 Yatham LN, Kennedy SH, Parikh SV, et al. Canadian Network for Mood and Anxiety Treatments (CANMAT) and International Society for Bipolar Disorders (ISBD) collaborative update of CANMAT guidelines for the management of patients with bipolar disorder: update 2013. Bipolar Disord 2013;15:1-44.

4 Kennedy SH, Lam RW, Parikh SV, etal. Canadian Network for Mood and Anxiety Treatments (CANMAT) clinical guidelines for the management of major depressive disorder in adults. Introduction. J Affect Disord 2009;117(suppl 1):S1-2.

5 Viguera AC, Whitfield T, Baldessarini RJ, et al. Risk of recurrence in women with bipolar disorder during pregnancy: prospective study of mood stabilizer discontinuation. Am J Psychiatry 2007;164:1817-24; quiz 923.

6 Gentile S. Antipsychotic therapy during early and late pregnancy. A systematic review. Schizophr Bull 2010:36:518-44.

7 Newcomer JW. Antipsychotic medications: metabolic and cardiovascular risk. / Clin Psychiatry 2007;68(suppl 4):8-13.

8 Barbui C, Conti V, Cipriani A. Antipsychotic drug exposure and risk of venous thromboembolism: a systematic review and meta-analysis of observational studies. Drug Safety 2014;37:79-90.

9 Abel KM. Fetal antipsychotic exposure in a changing landscape: seeing the future. Br I Psychiatry 2013:202:321-3.

10 Kaaja RJ, Greer IA. Manifestations of chronic disease during pregnancy. JAMA 2005;294:2751-7.

11 Levy AR, O'Brien BJ, Sellors C, et al. Coding accuracy of administrative drug claims in the Ontario drug benefit database. Can I Clin Pharmacol 2003;10:67-71.

12 Juurlink DPC, Croxford R, Chong A, et al. Canadian Institute for Health Information Discharge Abstract Database: A Validation Study. Toronto, 2006.

13 Hirdes JP, Smith TF, Rabinowitz T, et al. The Resident Assessment Instrument-Mental Health (RAl-MH): inter-rater reliability and convergent validity. J Behav Health Serv Res 2002;29:419-32.

14 Urbanoski KA, Mulsant BH, Willett P, et al. Real-world evaluation of the Resident Assessment Instrument-Mental Health assessment system. Can J Psychiatry 2012;57:687-95.

15 Hux JE, Ivis F, Flintoft V, et al. Diabetes in Ontario: determination of prevalence and incidence using a validated administrative data algorithm. Diabetes Care 2002:25:512-6.

16 Quan H, Khan N, Hemmelgarn BR, et al. Validation of a case definition to define hypertension using administrative data. Hypertension 2009:54:1423-8.

17 Parkerson GR Jr, Harrell FE Jr, Hammond WE, et al. Characteristics of adult primary care patients as predictors of future health services charges. Med Care 2001;39:1170-81.

18 You JJ, Alter DA, Stukel TA, et al. Proliferation of prenatal ultrasonography. CMA/ 2010;182:143-51.

19 Ray JG, Schull MJ, Urquia ML, et al. Major radiodiagnostic imaging in pregnancy and the risk of childhood malignancy: a population-based cohort study in Ontario. PLoS Med 2010;7:e1000337.
20 Gomes T, Juurlink DN, Ho JM, et al. Risk of serious falls associated with oxybutynin and tolterodine: a population based study. JUrol 2011;186:1340-4.

21 Schneeweiss S, Rassen JA, Glynn RJ, et al. High-dimensional propensity score adjustment in studies of treatment effects using health care claims data. Epidemiology 2009:20:512-22.

22 Rassen JA, Glynn RJ, Brookhart MA, et al. Covariate selection in high-dimensional propensity score analyses of treatment effects in small samples. Am J Epidemiol 2011;173:1404-13.

23 Ros H. Preeclampsia and other circulatory diseases during pregnancy-etiological aspects and impact on female offspring. Karolinska University Press, 2001.

24 Joseph KS, Fahey J. Validation of perinatal data in the Discharge Abstract Database of the Canadian Institute for Health Information. Chronic Dis Can 2009;29:96-100.

25 Joseph KS, Liu S, Rouleau J, et al. Severe maternal morbidity in Canada, 2003 to 2007: surveillance using routine hospitalization data and ICD-10CA codes. J Obstet Gynaecol Can 2010;32:837-46.

26 Ray JG, Sgro M, Mamdani MM, et al. Birth weight curves tailored to maternal world region. J Obstet Gynaecol Can 2012:34:159-71.

27 Crowther CA, Hiller JE, Moss JR, et al. Effect of treatment of gestational diabetes mellitus on pregnancy outcomes. $N$ Engl J Med 2005:352:2477-86

28 Ray JG, Vermeulen MJ, Shapiro JL, et al. Maternal and neonatal outcomes in pregestational and gestational diabetes mellitus, and the influence of maternal obesity and weight gain: the DEPOSIT study. Diabetes Endocrine Pregnancy Outcome Study in Toronto. Q/M 2001;94:347-56.

29 Landon MB, Spong CY, Thom E, et al. A multicenter, randomized trial of treatment for mild gestational diabetes. N Engl J Med 2009:361:1339-48

30 Landon MB, Mele L, Spong CY, et al. The relationship between maternal glycemia and perinatal outcome. Obstet Gynecol 2011;117:218-24

31 Urquia ML, Frank JW, Glazier RH, et al. Birth outcomes by neighbourhood income and recent immigration in Toronto. Health Reports 2007:18:21-30.

32 Canadian Perinatal Surveillance System. Perinatal health indicators for Canada: a resource manual. Ottawa, 2000.

33 Public Health Agency of Canada. Canadian Perinatal Health Report, 2008 Edition. Ottawa, Ontario, Canada, 2008.

34 Ross LE, Grigoriadis S, Mamisashvili L, et al. Selected pregnancy and delivery outcomes after exposure to antidepressant medication: a systematic review and meta-analysis. JAMA Psychiatry 2013;70:436-43

35 Boden R, Lundgren M, Brandt L, et al. Risks of adverse pregnancy and birth outcomes in women treated or not treated with mood stabilisers for bipolar disorder: population based cohort study. BMJ 2012;345:e7085

36 Hernandez-Diaz S, Smith CR, Shen A, et al. Comparative safety of antiepileptic drugs during pregnancy. Neurology 2012;78:1692-9.

37 Bellantuono C, Tofani S, Di Sciascio G, et al. Benzodiazepine exposure in pregnancy and risk of major malformations: a critical overview. Gen Hosp Psychiatry 2013;35:3-8.

38 Quispel C, Bangma M, Kazemier BM, et al. The role of depressive symptoms in the pathway of demographic and psychosocial risks to preterm birth and small for gestational age. Midwifery 2014;30:919-25.

39 Borders AE, Grobman WA, Amsden LB, et al. Chronic stress and low birth weight neonates in a low-income population of women. Obstet Gynecol 2007:109:331-8.

40 Reis M, Kallen B. Maternal use of antipsychotics in early pregnancy and delivery outcome. J Clin Psychopharmacol 2008;28:279-88.

41 Boden R, Lundgren M, Brandt L, et al. Antipsychotics during pregnancy: relation to fetal and maternal metabolic effects. Arch Gen Psychiatry 2012;69:715-21.

42 National Diabetes Surveillance System. Diabetes in Canada. Canada, 2008.

43 Canadian Chronic Disease Surveillance System. Hypertension in Canada, 2010.

(c) BMJ Publishing Group Ltd 2015

Supplementary tables S1 to S5 